\title{
Intermolecular control of thermoswitching and photoswitching phenomena in two spin-crossover polymorphs
}

\author{
M. Buron-Le Cointe,${ }^{1}$ J. Hébert, ${ }^{1}$ C. Baldé,${ }^{1,2,{ }^{*}}$ N. Moisan, ${ }^{1}$ L. Toupet,${ }^{1}$ P. Guionneau, ${ }^{2}$ \\ J. F. Létard,,$^{2, \dagger}$ E. Freysz, ${ }^{3}$ H. Cailleau, ${ }^{1}$ and E. Collet ${ }^{1, \dagger}$ \\ ${ }^{1}$ Institut de Physique de Rennes, Université de Rennes 1-CNRS, UMR 6251, Bâtiment 11A, Campus de Beaulieu, 35042 Rennes, France \\ ${ }^{2} I C M C B, C N R S$-Université de Bordeaux, 87 Av. du Doc. A. Schweitzer, 33608 Pessac, France \\ ${ }^{3}$ Laboratoire Onde et Matière d'Aquitaine, Université Bordeaux I-CNRS, UMR 5798, 33405 Talence, France
}

(Received 7 November 2011; published 21 February 2012)

\begin{abstract}
We discuss here the important role of intermolecular coupling for the thermal- and light-induced molecular state switching in the solid state. Investigations were performed on the two crystalline polymorphs of the spin-crossover $\left[\mathrm{Fe}-(\mathrm{PM}-\mathrm{BIA})_{2}(\mathrm{NCS})_{2}\right]$ material. In addition to structural studies at thermal equilibrium, lightinduced phenomena were investigated through photocrystallography, photomagnetic, and dynamical optical measurements. Strong similarities between the thermal-equilibrium and the out-of-equilibrium light-induced transformations are observed in each polymorph: strong cooperative phenomena in one polymorph versus weak cooperative ones in the second polymorph. These different responses of the two crystalline forms of the compound to external perturbations are discussed at the microscopic level in terms of Ising-like model and two-mode description of on-site molecular potentials.
\end{abstract}

\section{INTRODUCTION}

Molecular bistability in the solid state occurs when interacting molecules may be cooperatively switched between two different physical states under the influence of various control parameters. Such phenomena ${ }^{1,2}$ promise new routes for molecular optical switches or memory devices. This is exemplified by spin crossover compounds ${ }^{3-5}$ where cooperative switching between low-spin (LS) and high-spin (HS) molecular states is governed by elastic interactions in relation with the change of cell volume. ${ }^{6}$ An exceptional feature is the possibility of exhibiting such a switching both at thermal equilibrium, under control of temperature or pressure, as well as far away from equilibrium under control with light irradiation. ${ }^{7,8}$ It gives a nice opportunity to look into the interrelation between the physics of phase transition and that of nonlinear dynamics because these are the same cooperative interactions, that govern the stability of macroscopic states both at thermal equilibrium and out of equilibrium. The approach presented here, balancing the intra and intermolecular aspects involved during both thermal and photoconversions, can also be used in other bistable magnetic systems such as polycyanometallate derivatives, ${ }^{9-11}$ valence tautomeric complexes, ${ }^{12,13}$ ligand isomerization effects,,${ }^{14}$ etc.

The spin-state switching can occur in molecular solids ${ }^{5}$ made of a metal ion located in a nearly octahedral ligand molecular field. It manifests itself by the change of magnetic and optical properties. In addition to the change of electronic state, intramolecular and intermolecular structural reorganization take place, especially the change of molecular geometry and unit cell volume. In the case of a $\mathrm{Fe}^{\mathrm{II}}$ atom $\left(3 d^{6}\right)$, the two possible electronic distributions among the $3 d$ split orbitals, correspond to the low spin (LS, $S=0$ ) or high spin (HS, $S=$ 2) molecular states. The competition between the electron pairing energy, the energy splitting between the $t_{2 g}$ and $e_{g}$ orbitals, the structural reorganization, and the entropy effects are balancing the stability of the LS and HS states at thermal equilibrium..$^{5,15}$ This family of tuneable magnetic systems is referred in the literature as spin crossover (SCO). ${ }^{5}$ Studies of the photoinduced phenomena on metal transition compounds mainly concern the effect of continuous laser excitation at low temperature ${ }^{16-18}$ - the so-called light induced excited spin-state trapping (LIESST) ${ }^{19}$ — with the recent introduction of pulsed laser effects. ${ }^{20-27}$ Under continuous light irradiation, the flow of energy from photon flux competes with the dissipation in the thermal bath and determines the stable steady states. It may give rise to hysteretic response under the effect of control parameter such as temperature (LITH, light-induced thermal hysteresis), ${ }^{28}$ pressure (LIPH, light-induced pressure hysteresis), ${ }^{29}$ or light excitation density ( $\mathrm{LIOH}$, light-induced optical hysteresis). ${ }^{30,31}$

There are few SCO crystals for which the spin crossover is associated with symmetry breaking at thermal equilibrium ${ }^{32-35}$ or in the photoinduced state ( ${ }^{36-38}$ and references therein). For most of the SCO systems, the spin-state switching occurs in one step without any symmetry change, as it is the case for the two polymorphs studied here. Then the physical picture is analogous to the liquid-gas phase transition and $n_{\mathrm{HS}}$, the HS fraction of molecules in the HS state, plays the same role as the density does in the liquid-gas transition. ${ }^{29}$ Depending on the strength of cooperative interactions, the spin-state switching, both at thermal equilibrium and out of equilibrium, can be gradual or discontinuous, i.e., a supercritical crossover or a first-order phase transition. In the second case, macroscopic bistability occurs around the transition temperature and it may give rise to a thermal hysteresis. It is of fundamental interest to test in which way non-equilibrium photosteady states, with the photon flux as control parameter, may present a universal behavior similar to that at thermal equilibrium, in particular from gradual to bistable one. This paper addresses this issue through the comparison of dynamical behavior after light irradiation with equilibrium spin-state switching in two polymorphs of the same molecular complex, [Fe-(PM$\mathrm{BIA})_{2}(\mathrm{NCS})_{2}$ ]: ${ }^{28,31,39-41}$ a first one with an orthorhombic 


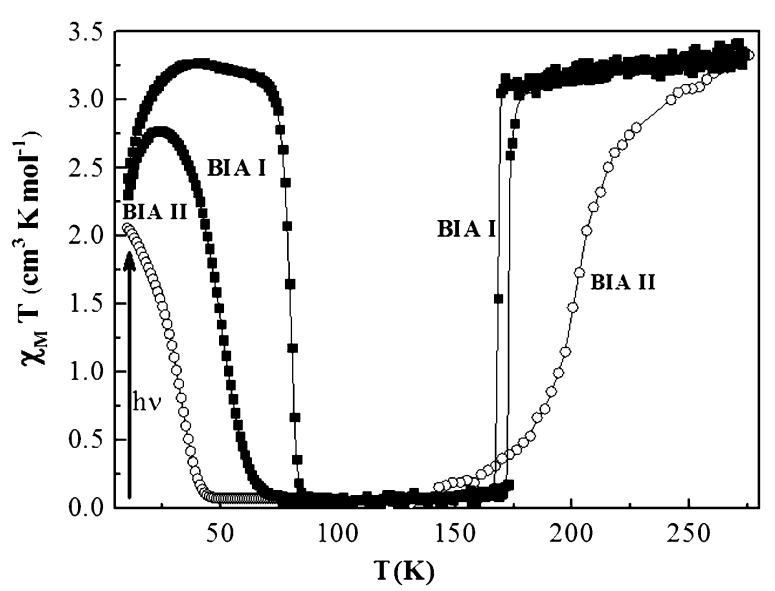

FIG. 1. Temperature dependence of the magnetic susceptibility times the temperature, $\chi_{M} T$, on cooling and heating modes for the two polymorphs of [Fe-(PM-BIA $\left.)_{2}(\mathrm{NCS})_{2}\right]$ (BIA I and BIA II). For BIA I, the first-order transition occurs around $170 \mathrm{~K}$ with a thermal hysteresis of $\approx 5 \mathrm{~K}$ width for BIA I $(\mathbf{\square})$. Under continuous laser irradiation (5 $\mathrm{mW} \cdot \mathrm{cm}^{-2}$ at $830 \mathrm{~nm}$ ), the HS state can be photoinduced (arrow) and a quasistatic light-induced thermal hysteresis (LITH) appears between cooling and warming modes ( $\boldsymbol{\square})$. For BIA II (o), a thermal crossover is centered around $205 \mathrm{~K}$ and no LITH is observed at low temperature.

crystal symmetry noted, hereafter BIA I, and a second one with a monoclinic crystal symmetry noted BIA II (see Fig. 1).

Sec. II presents the experimental details and procedure. In section III, we detail the structural data obtained both at thermal equilibrium and under $\mathrm{cw}$ laser excitation (photosteady states). Sec. IV is devoted to the mechanisms involved in the photo-switching under cw light irradiation both for the LS to HS conversion and for the relaxation. The relaxation mechanisms after a laser pulse excitation are also discussed through optical reflectivity measurements. Then results obtained on the two polymorphs are discussed at the microscopic level in the frame of Ising-like model and two-mode description of on-site molecular potentials (see Sec. V).

\section{EXPERIMENTAL}

\section{A. X-ray diffraction}

Structural investigations at thermal equilibrium and under continuous light irradiation were performed by x-ray diffraction on single crystals. Complete crystallographic data were collected both at thermal equilibrium at different temperatures and under light irradiation at low temperature $(\lambda=808$ $\mathrm{nm}$, with excitation densities up to $320 \mathrm{~mW} \cdot \mathrm{cm}^{-2}$ for BIA II at $15 \mathrm{~K}, 800 \mathrm{~mW} \cdot \mathrm{cm}^{-2}$ for BIA I at 15 and $80 \mathrm{~K}$ ). Laser was switched off during data collections in BIA I at $15 \mathrm{~K}$, since the photoinduced state has very long lifetime, ${ }^{28}$ but was kept on for BIA II to avoid spin-state relaxation. Structure of the photosteady HS state at $80 \mathrm{~K}$ in BIA I was obtained by trapping the HS state by flash cooling under laser irradiation and light was kept on during measurements. Data were collected on a four-circle Oxford Diffraction Xcalibur 3 diffractometer $\left(\mathrm{MoK}_{\alpha}\right.$ radiation) with a 2D Sapphire 3 CCD detector, on samples with typical sizes around $300 \times$ $100 \times 100 \mu \mathrm{m}^{3}$. The single crystals were mounted either in an Oxford Diffraction Helijet helium-flow cryostat, allowing reaching $15 \mathrm{~K}$ or in an Oxford Cryosystems nitrogen-flow cryostat allowing a better control of the temperature down to $78 \mathrm{~K}$. The unit-cell parameters and the data reduction were obtained with CrysAlis software from Oxford Diffraction. ${ }^{42}$ The structures were solved with SIR- $97^{43}$ and refined with SHELXL. ${ }^{44}$ Typical results of the structure refinement of the stable and photoinduced states gave final R1 factor, $0.033<$ $R 1<0.058 .{ }^{45}$

\section{B. Photomagnetic measurements}

The photomagnetic measurements were performed using a Spectra Physics Series $2025 \mathrm{Kr}+$ laser $\lambda=830 \mathrm{~nm}^{28}$ ) coupled via an optical fiber to the cavity of an MPMS-55 Quantum Design SQUID magnetometer. The optical power at the surface was adjusted between 8 and $28 \mathrm{~mW} \cdot \mathrm{cm}^{-2}$. Our previously published standardized method for measuring the $T$ (LIESST) temperature was followed, ${ }^{46,47}$ i.e., the sample was first cooled slowly to $10 \mathrm{~K}$ and then irradiated until the photosaturation. The laser was then switched off and the temperature increased at a rate of $0.3 \mathrm{~K} \cdot \mathrm{min}^{-1}$.

\section{Optical reflectivity measurements}

For measuring the dynamical optical response to pulsed laser excitation, BIA I was irradiated with a white light continuum. The light reflected by the sample was transmitted through a spectrometer at a given wavelength and recorded by a photomultiplier. The sample, a powder composed of microcrystallites (a few microns in radius) of BIA I, was sandwiched between two optical windows and was placed into an Oxford Optistat cryostat at $140 \mathrm{~K}$. The specular light reflected by the sample was collected and send through a $150 \mathrm{~mm}$ Jarrel-Ash spectrometer set to select the wavelength centered at $600 \mathrm{~nm}$. The resolution of the spectrometer was set to be $\sim 2 \mathrm{~nm}$. At the exit of the spectrometer the light was collected by a photomultiplier connected to an one-Mega-Ohms load. To excite the sample, a single laser pulse (Q-switched nanosecond frequency doubled $\mathrm{Nd}^{3+}$ :YAG laser, $\lambda=532 \mathrm{~nm}$, pulse width $8 \mathrm{~ns}$ ) was focused on a spot of about $3 \mathrm{~mm}$ in diameter. The laser pulse energy density on the sample was about $14 \mathrm{~mJ} \cdot \mathrm{cm}^{-2}$. Only a small fraction of light reflected by the sample at $600 \mathrm{~nm}$ within the central laser spot area was imaged and recorded by the spectrometer. The nanosecond laser drives the LS to HS switching and triggers the electronic device that measures the dynamical reflectivity.

For BIA II, the dynamical optical response was obtained on a single crystal by measuring on a fast oscilloscope the evolution of the $\mathrm{cw}$ reflectivity at $658 \mathrm{~nm}$ after a femtosecond laser excitation at $800 \mathrm{~nm}$ at $100 \mathrm{~K}$ (laser pulse energy density $\approx 10 \mathrm{~mJ} \cdot \mathrm{cm}^{-2}$ ). The measurements were accumulated in a stroboscopic way.

\section{DETAILED CRYSTALLOGRAPHIC ANALYSIS DURING THERMAL AND PHOTO-INDUCED CONVERSIONS}

The thermal and the cw light-induced spin conversions strongly differ in BIA I and BIA II, as already demonstrated by magnetic and photomagnetic measurements ${ }^{28,41}$ (see Fig. 1):(1) BIA I crystallizes in the orthorhombic space group 

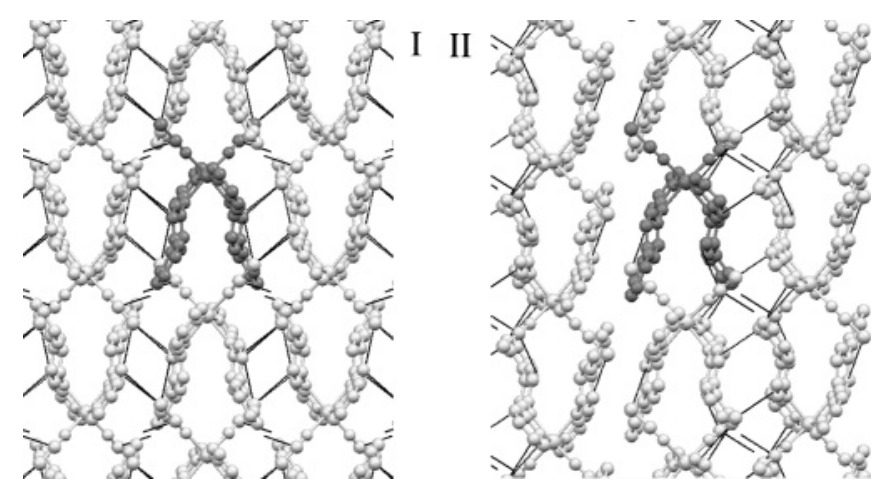

FIG. 2. Packing of the molecules in BIA I and BIA II polymorphs, with intermolecular contacts (lines) shorter than the sum of van der Waals radii at room temperature. For BIA I (left), the projection is done parallel to $a$ unit-cell axis. The $b$ unit-cell axis is horizontal and the $c$ axis is vertical. For BIA II (right), the projection is done parallel to $b$ unit-cell axis; the $c$ unit-cell axis is vertical. The dimerized layered structure of BIA II is observed along a direction for $\mathrm{T}>$ $250 \mathrm{~K}$ as well as in the photoinduced HS state at low temperature.

$P c c n^{28,40,41}$ with lattice parameters $a=12.962$ (5) $\AA, b=$ 15.223 (5) $\AA, c=17.644$ (52) $\AA$, and $V=3482$ (2) $\AA^{3}$ at $300 \mathrm{~K}$. It presents a temperature-induced phase transition with a $\approx 5 \mathrm{~K}$ width hysteresis around $T_{0} \approx 170 \mathrm{~K}$ and LITH at low temperature. ${ }^{31,48} T$ (LIESST) is defined as the limit temperature above which the HS state, photoinduced at $10 \mathrm{~K}$, has thermally relaxed using a heating rate of $0.3 \mathrm{~K} \cdot \mathrm{min}^{-1} 37$ and it is equal to $78 \mathrm{~K}$ for BIA I. ${ }^{28,41}$ (2) BIA II crystallizes in the monoclinic space group $P 2_{1} / c^{40,41,49}$ with $a=$ 17.548 (2) $\AA, b=12.591$ (1) $\AA, c=17.338$ (2) $\AA, \beta=$ $115.62(1)^{\circ}, V=3454.1(6) \AA^{3}$ at $295 \mathrm{~K}$. At thermal equilibrium, it presents a spin crossover extending from about 240 to $190 \mathrm{~K}$ and photoinduced effects are observed below $T($ LIESST) $=35 \mathrm{~K}$ without any hysteresis phenomenon (see Fig. 1). ${ }^{41}$

The molecular packing of BIA I and BIA II differ because of the different space groups, as presented in Fig. 2. We present in Fig. 3 the crystallographic signatures of the spin conversion with the geometric evolution of the first coordination sphere around $\mathrm{Fe}$ atom and the unit-cell parameters. The thermal conversion of the spin state, as well as the photoinduced conversion around $20 \mathrm{~K}$ for BIA II and up to $78 \mathrm{~K}$ for BIA I are clearly observed through the structural reorganization (see Fig. 4). At the intra-molecular level, the most important changes occur around the central $\mathrm{Fe}$ atom and the six $\mathrm{N}$ atoms bonding it to the ligand. As it is observed for other complexes of iron (II), a contraction of $\approx 10 \%$ of the average Fe-N bond length occurs between the HS and the LS states: $\langle\mathrm{Fe}-\mathrm{N}\rangle_{\mathrm{HS}}=$ $2.17(1) \AA$ and $\langle\mathrm{Fe}-\mathrm{N}\rangle_{\mathrm{LS}}=1.96(1) \AA$. This contraction is known to be associated with the electronic population of more bonding states in the LS electronic distribution, as observed by electron density analysis. ${ }^{50}$ Therefore the two polymorphs have the same average $\mathrm{Fe}-\mathrm{N}$ bonds in the two HS (high-temperature or photoinduced) and LS states. Differences are observed by looking at the deformation of the Fe- $\mathrm{N}_{6}$ octahedron, through the angular distortion parameter $\Sigma^{39,51}$ and on the length distortion parameter $\zeta$ [see Figs. 3(b) and $3(\mathrm{c})]$ : indeed if deformations from the perfect octahedron

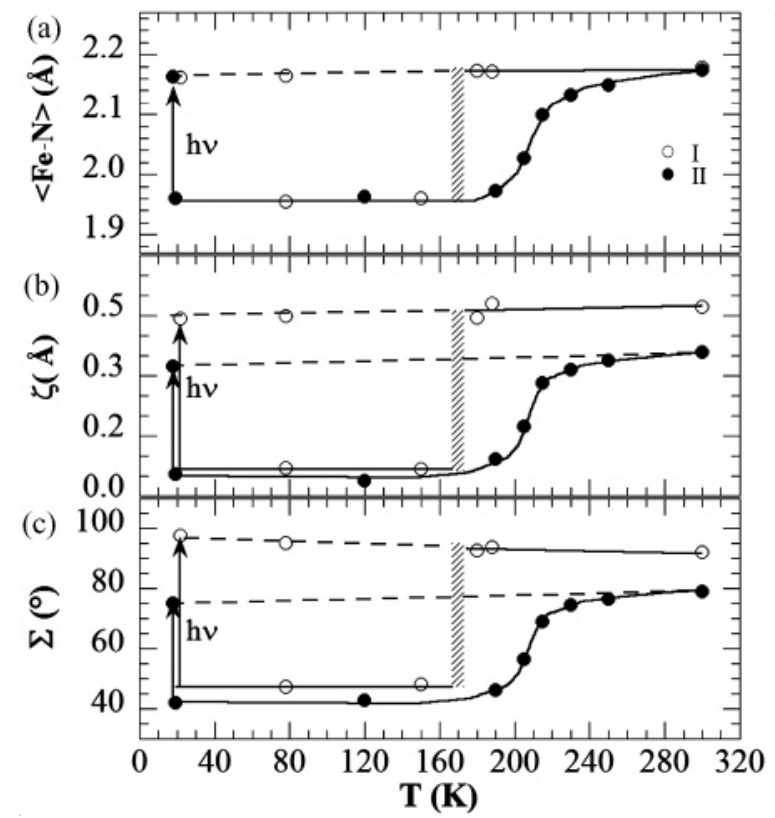

FIG. 3. (top) Average bonding Fe-N lengths $(\AA)$, (middle) deviation from this average value defined as $\zeta=\sum_{i=1}^{6}\left|\left(\mathrm{Fe}-\mathrm{N}_{i}\right)-\langle\mathrm{Fe}-\mathrm{N}\rangle\right|$ $(\AA)$, and (bottom) distortion of the $\mathrm{FeN}_{6}$ octahedron measured from the $\Sigma$ parameter, that is the sum of the 12 cis $\phi$ angles in the coordination sphere: $\sum=\sum_{i=1}^{12}\left|90-\phi_{i}\right|$ as a function of temperature

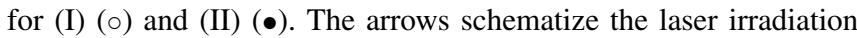
made $(\lambda=808 \mathrm{~nm})$ at $18 \mathrm{~K}$. Dashed lines are guides to the eye.

geometry are similar and small in the LS state, the molecular HS state of BIA I appears significantly more distorted than the one of BIA II, both for the HS stable state above $170 \mathrm{~K}$ and the photoinduced one at low temperature. These different deformations of the first coordination sphere around the iron atom, also observed through the larger motion of the ligand (see Fig. 4), can be directly related to differences in the packing (as constituent molecules are the same). Note that the difference in the coordination sphere distortions of BIA I and BIA II at the spin state change estimated through the twist angles has already been used to evidence the direct correlation between the $T$ (LIESST) temperatures and the $\mathrm{Fe}^{\mathrm{II}} \mathrm{N}_{6}$ distortion in $\left[\mathrm{FeL}(\mathrm{NCS})_{2}\right]$ compounds. ${ }^{51}$ It was demonstrated that the larger the distortion is, the higher $T$ (LIESST) appears.

In the two polymorphs, intermolecular distances shorter than the sum of van der Waals radii are observed and are the direct signature of intermolecular contacts. These are along the three spatial directions but anisotropic. Moreover, in the HS state of BIA II above $250 \mathrm{~K}$ or in the photoinduced HS state at low temperature the intermolecular contacts form a dimerized layered structure (see Fig. 2). Three different types of short contacts can be distinguished: (i) hydrogen ones (C. ..H or S. ..H) present at all temperatures in the two polymorphs, (ii) S. . .C-H ones involving S atoms of the NCS ligand, and (iii) C...C contacts between aromatic cycles of different molecules. Values of intermolecular distances of type (ii) are given in Table I. It has been previously reported, by comparing values at room temperature in $\left[\mathrm{Fe}-(\mathrm{PM}-\mathrm{L})_{2}(\mathrm{NCS})_{2}\right]$ family compounds, that this last type of contacts is directly correlated to the abruptness of the spin conversion: this latter is more abrupt with the shortest intermolecular distance ${ }^{39}$. Our 

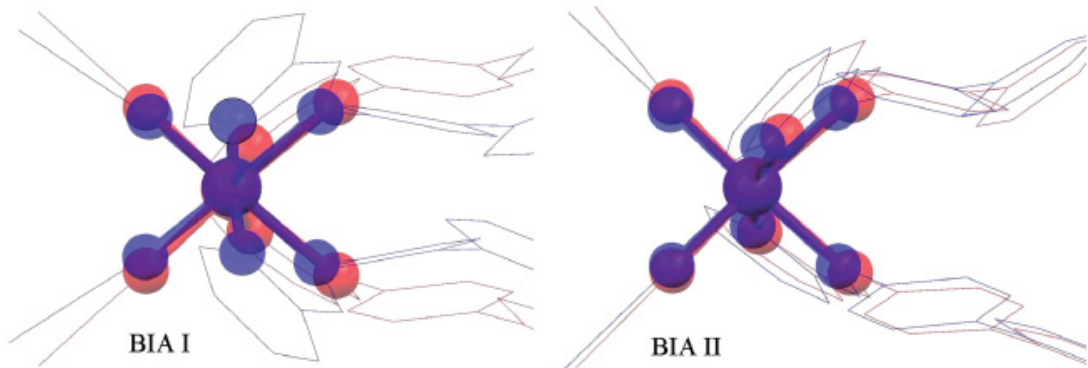

FIG. 4. (Color online) Structural deformation for the two polymorphs around the $\mathrm{FeN}_{6}$ octahedron between the LS (bleu) and HS (red) states. Central iron and six adjacent nitrogen atoms are drawn with thick lines. Thin lines represent the surrounding ligands and these are clearly sensitive to the molecular spin state change. extensive crystallographic studies at different temperatures show that S. . C-H distances are short contacts from the room temperature in BIA I, whereas it is the case only from 190 $\mathrm{K}$ down to low temperature in BIA II, when the system is mainly in the LS state. The third type of contacts, C. .C, have not been extensively detailed in literature before. These appear around $180 \mathrm{~K}$ in BIA I (3.366 $\AA$ at $180 \mathrm{~K}, 3.345 \AA$ at $150 \mathrm{~K}$, sum of van der Waals radii $=3.4 \AA$ ). In BIA II, these type of contacts already exist at room temperature inside the layers (which is $3.240 \AA$ ) and appear between the dimerized layers from $230 \mathrm{~K}$ (equals to $3.378 \AA$ ) and become stronger by lowering down the temperature (it is $3.367 \AA$ at $205 \mathrm{~K}$ for example). Thus these are short contacts of type (iii), which are involved in the change from dimerized layered structure at high-temperature to three-dimensional intermolecular network at low temperature in BIA II polymorph. The dimerized layered intermolecular arrangement is restored at $19 \mathrm{~K}$ in the photoinduced HS state. ${ }^{45}$

Concerning the unit-cell volume (see Fig. 5), an important thermal contraction is observed in both BIA I and BIA II polymorphs and BIA II is more compact than BIA I at all temperatures. Volume contractions resulting only from

TABLE I. Geometric data concerning the S. .C(H) interactions in the two polymorphs. PI denotes a photoinduced state. The sum of van der Waals radii are $3.5 \AA$ for S. . .C contacts.

\begin{tabular}{lccc}
\hline \hline & C...S $(\AA)$ & H...S $(\AA)$ & S...H-C $\left(^{\circ}\right)$ \\
\hline \multicolumn{4}{c}{ BIA I } \\
\hline$T=300 \mathrm{~K}$ & $3.430(5)$ & $2.869(5)$ & $120.0(2)$ \\
$T=180 \mathrm{~K}$ & $3.386(9)$ & $2.841(9)$ & $118.6(3)$ \\
$T=150 \mathrm{~K}$ & $3.455(4)$ & $2.839(4)$ & $124.7(2)$ \\
$T=80 \mathrm{~K}$ & $3.430(5)$ & $2.807(5)$ & $123.9(2)$ \\
$T=85 \mathrm{~K}, \mathrm{PI}$ & $3.445(9)$ & $2.805(9)$ & $125.6(9)$ \\
$T=15 \mathrm{~K}$, quenched & $3.352(4)$ & $2.829(4)$ & $116.7(2)$ \\
$T=15 \mathrm{~K}, \mathrm{PI}$ & $3.353(5)$ & $2.815(4)$ & $116.8(2)$ \\
& BIA II & & \\
$T=295 \mathrm{~K}$ & $3.539(5)$ & $2.860(5)$ & $130.7(2)$ \\
$T=290 \mathrm{~K}$ & $3.575(5)$ & $2.905(5)$ & $130.0(5)$ \\
$T=250 \mathrm{~K}$ & $3.542(5)$ & $2.877(5)$ & $129.5(2)$ \\
$T=230 \mathrm{~K}$ & $3.537(5)$ & $2.857(5)$ & $135.1(2)$ \\
$T=215 \mathrm{~K}$ & $3.522(5)$ & $2.850(5)$ & $130.2(2)$ \\
$T=205 \mathrm{~K}$ & $3.504(5)$ & $2.833(5)$ & $130.0(2)$ \\
$T=190 \mathrm{~K}$ & $3.483(4)$ & $2.794(4)$ & $131.7(2)$ \\
$T=120 \mathrm{~K}$ & $3.451(4)$ & $2.762(4)$ & $131.6(2)$ \\
$T=15 \mathrm{~K}$ & $3.452(4)$ & $2.767(4)$ & $131.2(2)$ \\
$T=15 \mathrm{~K}, \mathrm{PI}$ & $3.483(4)$ & $2.843(4)$ & $126.9(2)$ \\
\hline \hline
\end{tabular}

the change of spin states in the vicinity of $T_{1 / 2}$ region (excluding other thermal contraction effect) are similar for the two polymorphs: $\Delta V \approx 70 \AA^{3}$. This value was previously reported for BIA $\mathrm{II}^{49}$ and was obtained from the comparison with the temperature-dependent structural behavior of the cobalt complex (monoclinic $P 2_{1 / c}$ space group), [Co$\left.(\mathrm{PM}-\mathrm{BIA})_{2}(\mathrm{NCS})_{2}\right]$ that does not undergo a spin crossover. Intramolecular geometries and unit-cell volumes of the HS photoinduced state correspond to those observed at thermal equilibrium at high temperature within the thermal contraction in the two polymorphs. Moreover, in BIA I, structures of the photoinduced HS and of the thermally quenched states at helium temperatures do not differ significantly. ${ }^{45}$ This is in agreement with the conclusions previously drawn from the comparison between the thermally quenched HS state at $30 \mathrm{~K}$ and the room-temperature $\mathrm{HS}$ state in BIA I. ${ }^{52}$ Such similarities might be due to the absence of symmetry breaking during the first-order phase transition. The thermal hysteresis of BIA I is associated with growing of long-range domains of HS and LS phases, as demonstrated here by the coexistence of the corresponding Bragg peaks [see Fig. 6(a)]. This phase separation process is commonly observed in many first-order phase transitions. Differently, in BIA II, the gradual thermal conversion corresponding to a crossover from HS to LS is accompanied by a gradual shift of the Bragg peak in the reciprocal space [see Fig. 6(c)]. Therefore the different molecular packing in the two polymorphs result in a kind of internal pressure effect, which may shift the transition temperature and change the behavior from cooperative to crossover. The use of the term "pressure" is made on purpose since it has been demonstrated by diffraction measurements that when an external pressure of about $7 \mathrm{kbar}$ is applied on the BIA I polymorph, a structural phase transition from

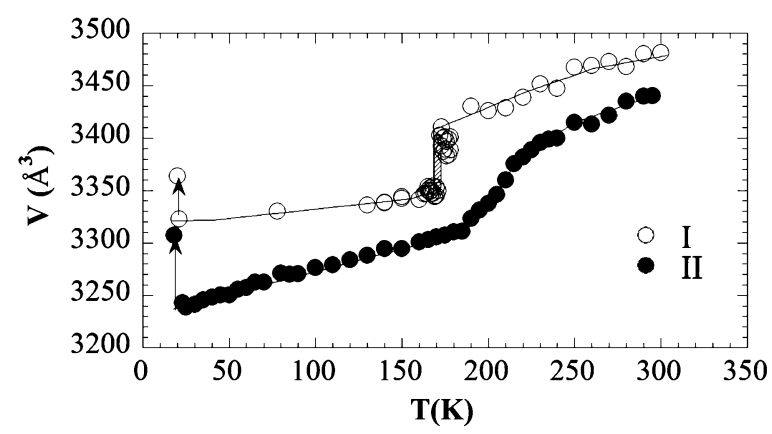

FIG. 5. Temperature evolution of the volume unit cell for (I) (o) and (II) $(\bullet)$. Lines are guides to the eye and arrows schematize the light irradiation $(\lambda=808 \mathrm{~nm})$. 


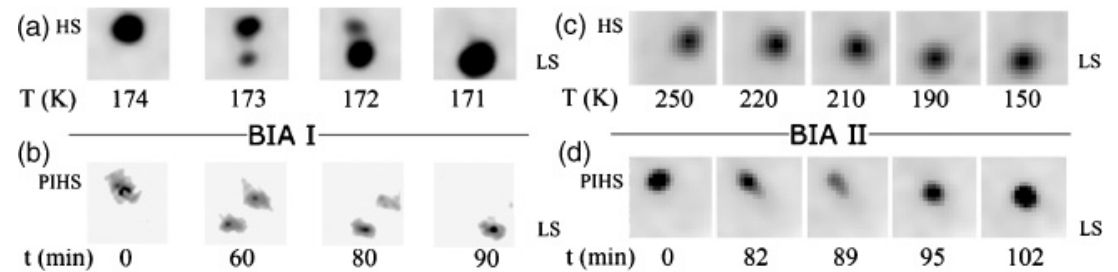

FIG. 6. Bragg peak shape and position evolution during the thermal HS to LS switching for BIA I (a) and BIA II (c) and during the relaxation from the photoinduced HS to LS states at $78 \mathrm{~K}$ for BIA I (b) and $20 \mathrm{~K}$ for BIA II (d).

the orthorhombic to the monoclinic symmetry occurs (space group $P 2_{1 / c}$ ), corresponding to the structural features of the BIA II polymorph. ${ }^{53}$

\section{COOPERATIVITY DURING THE PHOTOCONVERSIONS AND RELAXATIONS}

The stabilization of the metastable photoinduced HS state notably differs in the two polymorphs as illustrated by the $T$ (LIESST) values: $T$ (LIESST) $=78 \mathrm{~K}$ for BIA I but only $35 \mathrm{~K}$ for BIA II. The mechanisms leading to the macroscopic switching of the spin state as well as the relaxation ones ${ }^{41}$ are also very different in orthorhombic and monoclinic forms. The different responses of the polymorphs were tracked by measuring the magnetic responses under weak excitation densities $\left(8-28 \mathrm{~mW} \cdot \mathrm{cm}^{-2}\right)$, as presented in Figs. 7(a) and 7 (b), for $14 \mathrm{~mW} \cdot \mathrm{cm}^{-2}$. It indicates that the response of BIA I is lower once photoirradiation is started, but after $2000 \mathrm{~s}$ of laser excitation, the photoresponse strongly increases with respect to BIA II, indicating a self-accelerated mechanism characteristic of cooperative systems. Thus after 5 hours 30 minutes of irradiation, around $60 \%$ of the BIA I complex is switched to the HS state, whereas only $30 \%$ of the BIA II is converted. Using higher excitation densities, complete photoconversion can be easily achieved for both forms. As shown in Fig. 7(c), the threshold excitation density for reaching
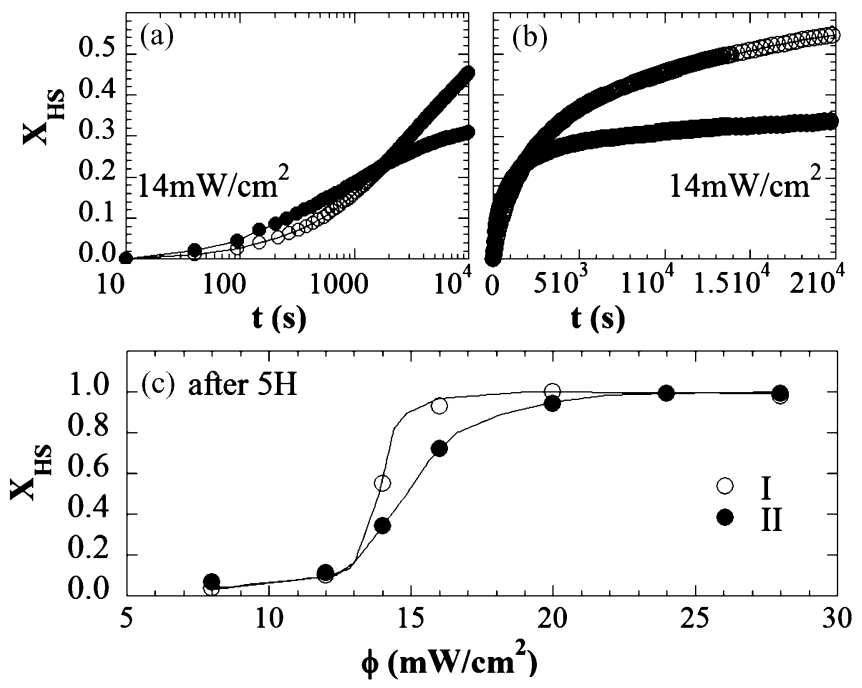

FIG. 7. (top) Photoinduced LS to HS conversion investigated by photomagnetic measurements at $10 \mathrm{~K}$ under low excitation density (around $14 \mathrm{~mW} \cdot \mathrm{cm}^{-2}$ ) for (I) (o) and (II) (•). The time needed for one measure gives the time resolution around $55 \mathrm{~s}$. (bottom) Photomagnetic response as a function of the excitation density for (I) (०) and (II) (•). a complete transformation of BIA I to the HS state after 5 hours of excitation is around $15 \mathrm{~mW} \cdot \mathrm{cm}^{-2}$. For BIA II, the response is more gradual with complete conversions requiring higher excitation densities, and here, again, the stronger cooperative effects in BIA I in comparison to BIA II are clear.

The relaxation dynamics from the HS state to the LS once laser excitation is stopped are also known to be different in the two polymorphs. ${ }^{41}$ It follows a nearly exponential law for the BIA II (not so different from the behavior characteristic of independent molecules relaxation), whereas a clear sigmoidal shape, characteristic of strong cooperative effects is observed for the BIA I. The present X-ray investigation of this mechanism by RX diffraction shows that this is due to the formation or not of separated HS and LS domains (see Fig. 6).

In BIA I, the phase separation mechanism with large domains formation is observed during the relaxation [see Fig. 6(b)], through the coexistence of Bragg peaks indexed in the HS or in the LS reciprocal lattices. This domain formation is similar to the phase separation process observed inside the thermal [see Fig. 6(a)] and the light-induced hysteresis reported in different system. ${ }^{31,32,34,41,54}$ In BIA II, a gradual and homogeneous variation of the spin state is associated with the progressive motion of the Bragg reflections from a purely HS lattice to a purely LS one [see Fig. 6(d)]; such a homogeneous relaxation was observed in another low cooperative spin crossover complex by neutron diffraction. ${ }^{55}$ The macroscopic relaxation mechanism is therefore similar to the ones observed during the thermal spin crossover for which a phase coexistence is observed for BIA I inside the thermal hysteresis, whereas a homogeneous state is observed for BIA II.

In these systems, the competition between light-induced population of the HS state and the thermally activated relaxation process to the LS state do not allow generating macroscopic HS states above $T$ (LIESST) with cw lasers. We could investigate the fastest relaxation mechanisms in BIA I and BIA II by generating a transient HS state with an intense and short laser pulse, and by measuring in the time domain optical reflectivity, also known to be sensitive to the change of electronic state. 5,20,21 This technique allows studying the relaxation after a pulse irradiation well above $T$ (LIESST), as presented here for both compounds at $[T(\mathrm{LIESST})+60 \mathrm{~K}$, i.e., $140 \mathrm{~K}$ for BIA I and $100 \mathrm{~K}$ for BIA II]. As shown in Fig. 8, the recovery to the LS thermal equilibrium state follows an exponential law for the form II whereas a clear sigmoidal shape is observed for the form I. It should also be noticed that the time necessary to completely recover the stable LS state after pulsed laser excitation decreases from hours below $T(\mathrm{LIESST})^{28,41}$ to $0.4 \mathrm{~s}$ for (I) at $140 \mathrm{~K}$ and $0.05 \mathrm{~s}$ for BIA II at $100 \mathrm{~K}$ (see Fig. 8). Thus there is one order of magnitude difference between the two polymorphs, which is here again due to the existence of strong cooperative effects in BIA I. 


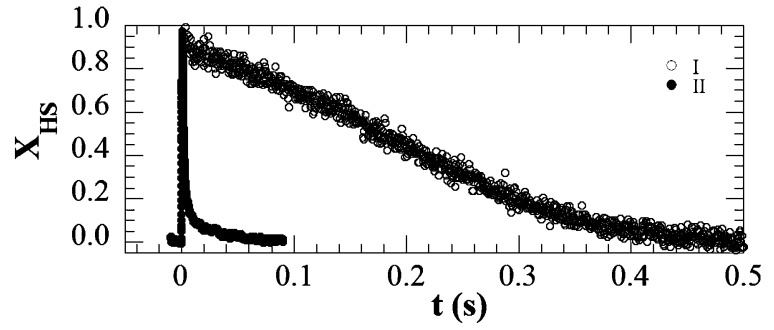

FIG. 8. Transformation rate deduced from reflectivity measurements. For each polymorph, experiments were performed around $60 \mathrm{~K}$ above $T$ (LIESST): $140 \mathrm{~K}$ for BIA I and $100 \mathrm{~K}$ for BIA II. Due to crystal cracking, a single-shot nanosecond experiment was performed on BIA I powder (ns pump $532 \mathrm{~nm}$, cw probe $600 \mathrm{~nm}$, $14 \mathrm{~mJ} \cdot \mathrm{cm}^{-2}$ ) whereas stroboscopic measurements were performed on a BIA II single crystal (fs pump $800 \mathrm{~nm}$, cw probe $658 \mathrm{~nm}$, $10 \mathrm{~mJ} \cdot \mathrm{cm}^{-2}$ ).

All the results presented above underline the strong similarities from the cooperative point of view between thermal equilibrium and out-of-equilibrium photoinduced processes following $\mathrm{cw}$ or pulsed laser excitation. The differences between BIA I and BIA II during out of equilibrium phototransformations and relaxations are to some extend the counterpart of cooperative effects observed at thermal equilibrium during thermoinduced spin transition in BIA I and spin crossover in BIA II as already observed on other compounds. ${ }^{56}$ It is of fundamental interest to discuss the origin of this difference of cooperativity for a deep understanding of the spin conversions in both cases. This task is addressed in the following section.

\section{ISING-LIKE MODEL AND TWO-MODE DESCRIPTION OF ON-SITE MOLECULAR POTENTIALS}

In the two BIA polymorphs, as in many spin-crossover complexes, the spin-state switching is an isostructural transformation, i.e., without any symmetry change as for the liquid-gas phase transition. The behavior of the HS concentration $n_{\mathrm{HS}}$ can be described in terms of an Ising-like model where the "field" is temperature dependent. 29,57,58 Indeed, fast electronic, including spin multiplicity, and vibrational degrees of freedom are integrated to obtain an effective Hamiltonian for the slower HS-LS molecular configuration degrees of freedom. The Hamiltonian is expressed as

$$
H=-h \sum_{i} S_{i}-J \sum_{\langle i, j\rangle} S_{i} S_{j},
$$

where $S_{i}$ is the Ising variable describing the two configuration states of the $i$ th molecule with eigenvalues +1 for HS and -1 for LS. Notice that the order parameter, which is the statistical average of the Ising variable $\langle S\rangle$ is directly related to the HS concentration since $\langle S\rangle=2 n_{\mathrm{HS}}-1$. The first part is the effective on-site Hamiltonian expressed in terms of a field $h(T)$, which depends linearly on temperature according to $h=-\left(\Delta-\frac{k_{B} T}{2} \ln g\right)$. The parameter $\Delta$ represents the enthalpy difference between HS and LS states, including isolated-molecule and crystal-field effects. The entropic effect is governed by $g$, the degeneracy ratio between the HS and LS states $\left(g=\frac{g_{\mathrm{HS}}}{g_{\mathrm{LS}}}\right)$, including vibrational and electronic multiplicities. Actually, the physical meaning of $h$ is the
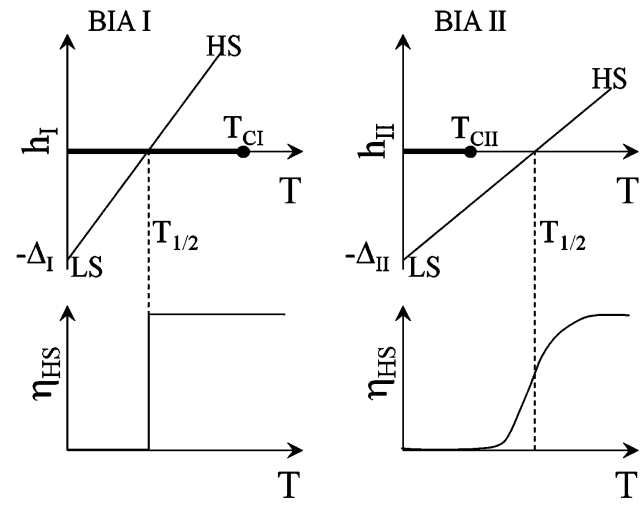

FIG. 9. (top) Schematic ( $h, T)$ phase diagram for BIA I (left) and for BIA II (right). (bottom) Schematic representation of the evolution of the HS fraction $n_{\mathrm{HS}}$ with temperature for BIA I (left) and for BIA II (right).

Gibbs energy difference per molecule between the fully HS and LS crystals, i.e., the chemical potential difference of the HS and LS species, $h=-\left(\frac{\Delta \mu}{2}\right)$. The second term of the Hamiltonian expresses the cooperative interactions in terms of the coupling constant $J$ between neighbors. Even if the elastic interactions yield to more complex pictures, ${ }^{59-61}$ this is the simplest way to express the cooperativity between molecules. Thermal spin state change behaviors can then be mapped in the universal $(h, T)$ phase diagram by an oblique line crossing the $h=0$ horizontal line, below (first-order phase transition) or above the critical temperature $T_{c}$ (supercritical crossover) (see Fig. 9). This crossing takes place at $T_{1 / 2}=$ $\frac{2 \Delta}{k_{B} \ln g}$, independent of the interaction parameter $J$, while in the mean-field approximation, the critical temperature $T_{c}$ is given by $T_{c}=\frac{p J}{k_{B}}$, where $p$ is the number of (equivalent) neighbors.

In the two studied polymorphs, differences come from the intermolecular contacts that are shorter and more numerous in BIA I than in BIA II (see Fig. 2 and Table I). Consequently, the coupling constant $J$ is larger in BIA I than in BIA II $\left(J_{\mathrm{I}}>J_{\mathrm{II}}\right)$ and therefore the temperatures of the corresponding critical points are different. As indicated in Fig. 9, $T_{c \mathrm{I}}>T_{c \mathrm{II}}$ since $T_{c}$ is proportional to $p J$ as discussed above. Secondly, effective fields $h$ are not identical in the two polymorphs. A significant contribution to the difference in $h$ values should come from the vibrational contribution to the degeneracy ratio $g$. It should be higher in BIA I than in BIA II since the molecular deformation is more important in BIA I than in BIA II (see Fig. 3), with, in particular, a significant contribution of the ligand (see Fig. 4) and then $g_{\mathrm{I}}>g_{\mathrm{II}}$. The effect of the difference of $\Delta$ values (enthalpy difference between HS and LS states) may be considered as smaller since only the crystal field can contribute. The experimental difference of $T_{1 / 2}$ values between BIA I (170 K) and BIA II (205 K) agrees well with this line of argument. As schematically represented in Fig. 9, the temperature-dependent fields for BIA I and BIA II reach

$h_{\mathrm{I}}=-\left(\Delta_{\mathrm{I}}-\frac{k_{B} T}{2} \ln g_{\mathrm{I}}\right)$ and $h_{\mathrm{II}}=-\left(\Delta_{\mathrm{II}}-\frac{k_{B} T}{2} \ln g_{\mathrm{II}}\right)$

with a higher slope for BIA I than for BIA II and $\Delta_{\mathrm{I}} \approx \Delta_{\mathrm{II}}$. Since $T_{c \mathrm{I}}>T_{c \mathrm{II}}$, both effects favor a first-order transition for BIA I and a crossover for BIA II. 
For describing the light-induced switching, the dynamics of this Ising-like model has to be considered. ${ }^{29,57,59,60}$ In the mean-field approach, this leads to a deterministic phenomenological evolution equation for the dynamical variable $n_{\mathrm{HS}}(t)$, which expresses the competition of photo-induced process and thermal recovery:

$$
d n_{\mathrm{HS}} / d t=d n_{\mathrm{HS}} / d t^{\text {light }}-d n_{\mathrm{HS}} / d t^{\text {thermal }} .
$$

This can also be written as

$$
d n_{\mathrm{HS}} / d t=\sigma I\left(1-n_{\mathrm{HS}}\right)-k\left(T, n_{\mathrm{HS}}\right) n_{\mathrm{HS}},
$$

where the light excitation term is written as proportional to the intensity $I$ of photo-irradiation (considering independent molecular response to photoexcitation) and the thermal term governing the relaxation $\mathrm{HS} \rightarrow \mathrm{LS}$ takes into account the cooperative interactions through a nonconstant kinetic coefficient function of $n_{\mathrm{HS}}$. Indeed, the medium is no more passive but active and the relaxation rate is depending from the environment. The larger is the number of LS species, the more accelerated is the relaxation process of the photo-induced HS molecules. This coefficient can be written $k\left(n_{\mathrm{HS}}\right)=k_{0}(T) \exp \left[\alpha(T)\left(1-n_{\mathrm{HS}}\right)\right]$ as empirically proposed by Hauser. ${ }^{62}$ The mean-field approach of the Ising-like dynamics allows justifying it by choosing an Arrhenius-like dynamics and expressing $\alpha$ as proportional to the coupling constant $J{ }^{29,57}$ Steady states are no more determined by a thermodynamic potential but by a dynamic potential $V\left(n_{\mathrm{HS}}\right)$. Steady states are achieved once the thermal recovery compensates the photo-induced process such as $d n_{\mathrm{HS}} / d t=0$, then for extrema of $V\left(n_{\mathrm{HS}}\right)$. A dynamic first-order-like phase transition line occurs in the control parameter $(I, T)$ plane below the critical point $C^{*}\left(I_{c^{*}}, \mathrm{~T}_{c^{*}}\right)$ corresponding to $n_{\mathrm{HS}}=\frac{1}{2}$ and $T_{c^{*}}=$ $\frac{T_{c}}{2}$. Below $C^{*}$, a light-induced optical hysteresis (LIOH) can be observed, while above this point the light transformation occurs gradually. Such an out-of-equilibrium dynamics is not limited to the spin-crossover systems discussed here. It is, for example, analogous to the one observed in alloys or complex materials under neutron irradiation. ${ }^{63}$ There is then a competition between irradiation-induced damaging and thermal recovery, which allows again defining the steady states.

In the two spin-crossover crystals presented here, all experimental observations under light irradiation show that light-induced transition takes place below the critical point $C_{\mathrm{BIAI}}^{*}$ in BIA I while the out-of-equilibrium photo-transformation occurs above $C_{\text {BIAII }}{ }^{*}$ in BIA II. Thus a $\mathrm{LIOH}$ is observed only in BIA I around $80 \mathrm{~K},{ }^{31}$ indicating that $T_{c^{*} \text { BIAI }}$ is higher than $80 \mathrm{~K}$. The different behaviors of BIA I and BIA II are also clearly visible on the initial dynamical response to cw excitation [see Figs. 7(a) and 7(b)]. The response of BIA I is smaller than the one of BIA II in the early stage, whereas it becomes higher above a critical value of photoexcited species. This behavior results from the dependence of the relaxation rate with the population of LS state [second term in Eqs. (2) and (3)]: in the cooperative BIA I system, a higher relaxation rate is observed when the fraction of LS molecule is important. ${ }^{41}$ Therefore in the initial stage, the relaxation of photoconverted HS molecules to LS state is faster than the photoconversion and the photoconverted molecular fraction is very low. In the less cooperative polymorph BIA II,
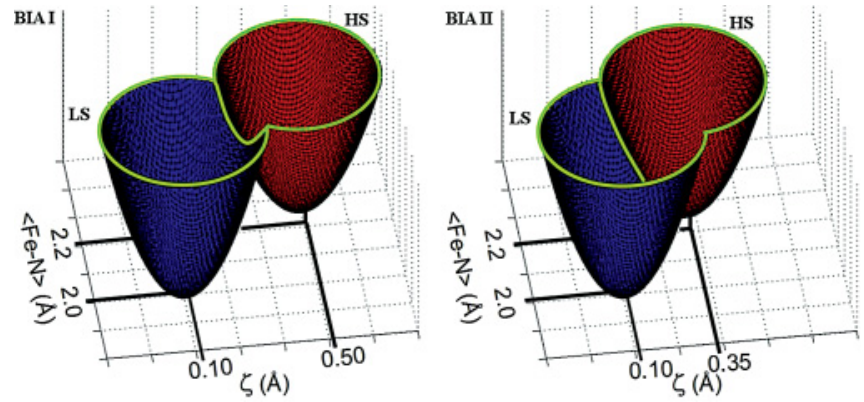

FIG. 10. (Color online) Two-mode description of on-site molecular potentials for BIA I and BIA II as a function of the average $\langle\mathrm{Fe}-\mathrm{N}\rangle$ bond length $(\AA)$ and of the length distortion parameter $\zeta=\sum_{i=1}^{6}\left|\mathrm{Fe}-\mathrm{N}_{i}-\langle\mathrm{Fe}-\mathrm{N}\rangle\right|(\AA)$.

the relaxation rate is nearly exponential, i.e., does not depend on the fraction of molecules in the HS state: in Eq. (2), the thermal recovery becomes nearly independent of $n_{\mathrm{HS}}$ when $k\left(T, n_{\mathrm{HS}}\right)$ is dominated by $k_{0}(T)$. Once the population of the HS state is above a critical value (around 20\%, Fig. 7), the relaxation rate becomes lower and lower for BIA I. For this reason, the photoconverted molecular fraction becomes higher in BIA I than in BIA II, as a result of the cooperative effect.

The different behaviors under light irradiation of the two polymorphs BIA I and BIA II are also visible on the $T$ (LIESST) values, $78 \mathrm{~K}$ for BIA I versus $35 \mathrm{~K}$ for BIA II. Such a more or less important stabilization of the low-temperature photoinduced HS state can be understood by considering a two-mode description of the on-site molecular potentials (see Fig. 10). In such a description, ${ }^{64}$ in addition to the well-known average Fe-N bond length change of $0.2 \AA$ between pure HS and LS states, ${ }^{5}$ the anisotropic deformation of the coordination sphere around $\mathrm{Fe}$ atom is taken into account via the deformation parameter (for example, $\zeta$ ). This last contribution is more important when the expansion of the coordination sphere around $\mathrm{Fe}$ is distorted. As this deformation is stronger in BIA I than in BIA II, it results in an increase of the energy barrier between the HS and LS states (see Fig. 10). Therefore the photoinduced HS is more easily stabilized at low temperature in BIA I than in BIA II, since the thermal energy necessary to overcome the energy barrier is larger in BIA I. Because molecules are identical in the two polymorphs, the more or less important deformation of the first coordination sphere around $\mathrm{Fe}$ is then directly related to intermolecular interactions. Shorter intermolecular contacts observed in BIA I within the orthorhombic arrangement of the molecules in BIA I favors larger molecular deformations in comparison to BIA II (monoclinic symmetry). As a result, the deformation of the coordination sphere can be considered as the microscopic origin of the increase of the $T$ (LIESST). ${ }^{51}$ A similar picture has also been used recently to discuss studies on a series of $\mathrm{Fe}(\mathrm{III})$ compounds with $\pi-\pi$ intermolecular interactions. ${ }^{65}$

\section{CONCLUSION}

Studies on two polymorphs of the [Fe-(PM-BIA $\left.)_{2}(\mathrm{NCS})_{2}\right]$ spin-crossover compound gave a nice opportunity to deepen the interrelation between the physics of phase transition at thermal equilibrium and the mechanisms driving out-of- 
equilibrium photoinduced processes, in the same molecular system with similar feedback mechanism originating from the similar intermolecular interactions. We observe here strong similarities from the cooperative point of view between behaviors at thermal equilibrium (phase transition in BIA I versus crossover in BIA II) and those under light irradiation: self-amplified (BIA I) versus quasilinear (BIA II) phototransformation, sigmoidal and phase separation relaxation process (BIA I) versus exponential and homogeneous one (BIA II). In that sense, studies at thermal equilibrium are essential to understand those under light irradiation. Photoinduced phenomena are interesting for applications in optoelectronic such as high-density memories, optical switches, etc., and efforts are underway for predicting photo-induced phenomena and in particular to anticipate on the $T$ (LIESST). The present study illustrates that not only the nature of the molecule but also its environment is an important parameter. Strong to weaker intermolecular interactions can make one spin-crossover system more or less cooperative with direct consequences on both the thermal spin-state conversion and the photoinduced phenomena under cw and pulsed light irradiation. Comparing polymorphs allows balancing relative contributions of intra and intermolecular degrees of freedom. This approach can be extended to other photoactive systems like polycyanometallate derivatives, valence tautomeric complexes, etc., also presenting photoconversions similar to thermal ones. By discriminating among different parameters, the ones playing the key role, such approach will lead to new possibilities for optimizing and controlling the response of materials to light excitation.

\section{ACKNOWLEDGMENTS}

This work was supported by Europe (FEDER), the Institut Universitaire de France, the National Research Agency (ANR09-BLAN-0212), the Région Bretagne (CREATE Ultimate 4146, PRIR Femtocom 2178, PhD funding for J. Hébert) and Rennes Metropole.
*Current address: Laboratoire de Chimie et Physique des Matériaux (LCPM) Université de Ziguinchor, Bp: 523 Ziguinchor, Sénégal.

†eric.collet@univ-rennes1.fr and letard@icmcb-bordeaux.cnrs.fr

${ }^{1}$ O. Sato, J. Tao, and Y. Z. Zhang, Angew. Chem. Int. Ed. 46, 2152 (2007).

${ }^{2}$ A. Bousseksou, G. Molnar, L. Salmon, and W. Nicolazzi, Chem. Soc. Rev. 40, 3313 (2011).

${ }^{3}$ O. Kahn and C. J. Martinez, Science 279, 44 (1998).

${ }^{4}$ P. Gütlich, Y. Garcia, and T. Woike, Coord. Chem. Rev. 219-221, 839 (2001).

${ }^{5}$ Spin Crossover in Transition Metal Compounds, edited by P. Gütlich and H. A. Goodwin, Top. Curr. Chem. 233-235 (Springer, New York, 2004).

${ }^{6}$ M. Nishino, K. Boukheddaden, Y. Konishi, and S. Miyashita, Phys. Rev. Lett. 98, 247203 (2007).

${ }^{7}$ Photoinduced Phase Transitions, edited by K. Nasu (World Scientific, Singapore, 2005).

${ }^{8}$ Yoshinori Tokura, J. Phys. Soc. Jpn. 75, 011001 (2006).

${ }^{9}$ H. W. Liu, K. Matsuda, Z. Z. Gu, K. Takahashi, A. L. Cui, R. Nakajima, A. Fujishima, and O. Sato, Phys. Rev. Lett. 90, 167403 (2003).

${ }^{10}$ A. Bleuzen, J. D. Cafun, A. Bachschmidt, M. Verdaguer, P. Münsch, F. Baudelet, and J. P. Itié, J. Phys. Chem. C 112, 17709 (2008).

${ }^{11}$ M. Nihei, Y. Sekine, N. Sugamani, K. Nakazawa, A. Nakao, H. Nakao, Y. Murakami, and H. Oshio, J. Am. Chem. Soc. 133, 3592 (2011).

${ }^{12}$ P. Dapporto, A. Dei, G. Poneti, and L. Sorace, Chem. Eur. J. 14, 10915 (2008).

${ }^{13}$ A. Dei, G. Poneti, and L. Sorace, Inorg. Chem 49, 3271 (2010).

${ }^{14}$ M. L. Boillot, S. Pillet, A. Tissot, E. Rivière, N. Claiser, and C. Lecomte, Inorg. Chem. 48, 4729 (2009).

${ }^{15}$ P. Gütlich, A. Hauser, and H. Spiering, Angew. Chem. 33, 2024 (1994)

${ }^{16}$ S. Decurtins, P. Gütlich, C. P. Köhler, H. Spiering, and A. Hauser, Chem. Phys. Lett. 105, 1 (1984).

${ }^{17}$ A. Hauser, Chem. Phys. Lett. 124, 543 (1986).
${ }^{18}$ A. Hauser, J. Jeftic, H. Romstedt, R. Hinek, and H. Spiering, Coord. Chem. Reviews 190-192, 471 (1999).

${ }^{19}$ J. F. Létard, L. Capes, G. Chastanet, N. Moliner, S. Létard, J. A. Real, and O. Kahn, Chem. Phys. Lett. 313, 115 (1999).

${ }^{20}$ C. Enachescu, A. Hauser, J. J. Girerd, and M. L. Boillot, Chem. Phys. Chem. 7, 1127 (2006).

${ }^{21}$ G. Galle, J. Degert, C. Mauriac, C. Etrillard, J. F. Létard, and E. Freysz, Chem. Phys. Lett. 500, 18 (2010).

${ }^{22}$ M. Lorenc, J. Hébert, N. Moisan, E. Trzop, M. Servol, M. Buron-Le Cointe, H. Cailleau, M. L. Boillot, E. Pontecorvo, M. Wulff, S. Koshihara, and E. Collet, Phys. Rev. Lett. 103, 028301 (2009).

${ }^{23}$ O. Fouché, J. Degert, G. Jonusauskas, N. Daro, J. F. Létard, and E. Freysz, Phys. Chem. Chem. Phys. 12, 3044 (2010).

${ }^{24}$ S. Bonhommeau, G. Molnar, S. Cobo, D. Ostrovskii, and A. Bousseksou, Polyhedron 28, 1610 (2009).

${ }^{25}$ N. Moisan, M. Servol, M. Lorenc, A. Tissot, M. L. Boillot, H. Cailleau, S. Koshihara, and E. Collet, C. R. Chimie J. 11, 1235 (2008).

${ }^{26}$ E. Collet, M. Lorenc, M. Cammarata, L. Guérin, M. Servol, A. Tissot, M. L. Boillot, H. Cailleau, and M. Buron-Le Cointe, Chem. Eur. J. 18, 2051 (2012).

${ }^{27}$ E. Collet, N. Moisan, C. Baldé, R. Bertoni, E. Trzop, C. Lauhlé, M. Lorenc, M. Servol, H. Cailleau, A. Tissot, M. L. Boillot, T. Graber, R. Henning, P. Coppens, and M. Buron-Le Cointe, Phys. Chem. Chem. Phys., doi:10.1039/C2CP23587K.

${ }^{28}$ J. F. Létard, P. Guionneau, L. Rabardel, J. A. K. Howard, A. E. Goeta, D. Chasseau, and O. Kahn, Inorg. Chem. 37, 4432 (1998).

${ }^{29}$ K. Boukheddaden, I. Shteto, B. Hôo, and F. Varret, Phys. Rev. B 62, 14806 (2000).

${ }^{30}$ A. Desaix, O. Roubeau, J. Jeftic, J. G. Haasnoot, K. Boukheddaden, E. Codjovi, J. Linares, M. Nogues, and F. Varret, Eur. Phys. J. B 6, 183 (1998).

${ }^{31}$ K. Ichiyanagi, J. Hébert, L. Toupet, H. Cailleau, P. Guionneau, J. F. Létard, and E. Collet, Phys. Rev. B 73, 060408(R) (2006). 
${ }^{32}$ P. Guionneau, F. Le Gac, S. Lakhloufi, A. Kaiba, D. Chasseau, J. F. Létard, P. Négrier, D. Mondieig, J. A. K. Howard, and J.-M. Léger, J. Phys.: Condens. Matter 19, 326211 (2007).

${ }^{33}$ D. Chernyshov, M. Hostettler, K. W. Törnroos, and H. B. Bürgi, Angew. Chem. Int. Ed. 42, 3825 (2003).

${ }^{34}$ N. Huby, L. Guérin, E. Collet, L. Toupet, J. C. Ameline, H. Cailleau, T. Roisnel, T. Tayagaki, and K. Tanaka, Phys. Rev. B 69, 020101 (2004).

${ }^{35}$ M. Buron-Le Cointe, N. Ould Moussa, E. Trzop, A. Moréac, G. Molnar, L. Toupet, A. Bousseksou, J.-F. Létard, and G. S. Matouzenko, Phys. Rev. B 82, 214106 (2010).

${ }^{36} \mathrm{~N}$. Bréfuel, H. Watanabe, L. Toupet, J. Come, M. Kojima, N. Matsumoto, E. Collet, K. Tanaka, and J. P. Tuchagues, Angew. Chem. Int. Ed. 48, 9304 (2009).

${ }^{37}$ C.-F. Sheu, S.-M. Chen, S.-C. Wang, G.-H. Lee, Y.-H. Liu, and Y. Wang, Chem. Commun. 7512 (2009); C. H. Shih, C. F. Cheu, K. Kato, K. Sugimoto, J. Kim, Y. Wang, and M. Takata, Dalton Trans. 39, 9794 (2010).

${ }^{38}$ N. Bréfuel, E. Collet, H. Watanabe, M. Kojima, N. Matsumoto, L. Toupet, K. Tanaka, and J. P. Tuchagues, Chem. Eur. J. 16, 14060 (2010).

${ }^{39}$ P. Guionneau, J. F. Létard, D. S. Yufit, D. Chasseau, G. Bravic, A. E. Goeta, J. A. K. Howard, and O. Kahn, J. Mater. Chem 9, 985 (1999).

${ }^{40}$ M. Marchivie, P. Guionneau, J. F. Létard, and D. Chasseau, Acta Cryst. B 59, 479 (2003).

${ }^{41}$ J. F. Létard, G. Chastanet, O. Nguyen, S. Marcen, M. Marchivie, P. Guionneau, D. Chasseau, and P. Gütlich, Monatshefte für Chemie 134, 165 (2003).

${ }^{42}$ CrysAlis RED, Oxford Diffraction Ltd, Version 1.171.32.5 (2007).

${ }^{43}$ A. Altomare, M. C. Burla, M. Camalli, G. Cascareno, C. Giacovazzo, A. Guagliardi, A. G. G. Moliterni, G. Polidori, and R. Spagna, J. Appl. Cryst. 32, 115 (1999).

${ }^{44}$ G. M. Sheldrick, Acta Cryst. A 64, 112 (2008).

${ }^{45} \mathrm{CCDC}-826900$ up to CCDC-826906 (BIA I) as well as CCDC827842 up to CCDC-827851 (BIA II) contain the supplementary crystallographic data for this paper. These data can be obtained free of charge via www.ccdc.cam.ac.uk/conts/retrieving.html (or from the Cambridge Crystallographic Data Centre, 12 Union Road, Cambridge CB21EZ, UK; fax: (+44) 1223-336033; or deposit@ccdc.cam.uk).
${ }^{46}$ J. F. Létard, J. Mater. Chem. 16, 2550 (2006).

${ }^{47}$ J. F. Létard, P. Guionneau, O. Nguyen, J. Sanchez Costa, S. Marcen, G. Chastanet, M. Marchivie, and L. Goux-Capes, Chem. Eur. J. 11, 4582 (2005).

${ }^{48}$ C. Enachescu, R. Tanasa, A. Stancu, G. Chastanet, J.-F. Létard, J. Linares, and F. Varret, J. Appl. Phys. 99, 08 J504 (2006).

${ }^{49} \mathrm{P}$. Guionneau, M. Marchivie, G. Bravic, J.-F. Létard, and D. Chasseau, J. Mater. Chem. 12, 2546 (2002).

${ }^{50}$ V. Legrand, S. Pillet, M. Souhassou, N. Lugan, and C. Lecomte, J. Am. Chem. Soc. 128, 13921 (2006).

${ }^{51}$ M. Marchivie, P. Guionneau, J.-L. Létard, and D. Chasseau, Acta Cryst. B 61, 25 (2005).

${ }^{52}$ M. Marchivie, P. Guionneau, J.-F. Létard, D. Chasseau, and J. A. K. Howard, J. Phys. Chem. Solids 65, 17 (2004).

${ }^{53}$ V. Legrand, F. Le Gac, P. Guionneau, and J.-F. Létard, J. Appl. Cryst 41, 637 (2008).

${ }^{54}$ S. Pillet, V. Legrand, M. Souhassou, and C. Lecomte, Phys. Rev. B 74, 140101 (2006)

${ }^{55}$ A. Goujon, B. Gillon, A. Debede, A. Cousson, A. Gukasov, J. Jeftic, G. J. McIntyre, and F. Varret, Phys. Rev. B 73, 104413 (2006).

${ }^{56}$ D. Glijer, J. Hébert, E. Trzop, E. Collet, L. Toupet, H. Cailleau, G. S. Matouzenko, H. Z. Lazar, J. F. Létard, S. Koshihara, and M. Buron-Le Cointe, Phys. Rev. B 78, 134112 (2008).

${ }^{57}$ K. Boukheddaden, I. Shteto, B. Hôo, and F. Varret, Phys. Rev. B 62, 14796 (2000).

${ }^{58}$ M. Nishino, S. Miyashita, and K. Boukheddaden, J. Chem. Phys. 118, 4594 (2003).

${ }^{59}$ C. Chong, F. Varret, and K. Boukheddaden, Phys. Rev. B 81, 014104 (2010).

${ }^{60}$ K. Boukheddaden, M. Nishino, and S. Miyashita, Phys. Rev. Lett. 100, 177206 (2008).

${ }^{61}$ M. Nishino, K. Boukheddaden, and S. Miyashita, Phys. Rev. B 79, 012409 (2009).

${ }^{62}$ A. Hauser, J. Jeftic, H. Romstedt, R. Hinek, and H. Spiering, Coord. Chem. Rev 190-192, 471 (1999).

${ }^{63}$ G. Martin and P. Bellon, C. R. Phys. 9, 323 (2008).

${ }^{64}$ A. Hauser, C. Enachescu, M. Lawson Daku, A. Vargas, and N. Amstutz, Coord. Chem. Rev. 250, 1642 (2006).

${ }^{65}$ S. Hayami, K. Hiki, T. Kawahara, Y. Maeda, D. Urakami, K. Inoue, M. Ohama, S. Kawata, and O. Sato, Chem. Eur. J. 15, 3497 (2009). 